\title{
Magnetic fields in multiple bright-rimmed clouds in different directions of H II region IC 1396 - II
}

\author{
Archana Soam ${ }^{1,3 \star}$, G. Maheswar ${ }^{2,3}$, Chang Won Lee ${ }^{1,4}$, Neha S..$^{3,5}$, Kee-Tae Kim ${ }^{1}$ \\ ${ }^{1}$ Korea Astronomy \& Space Science Institute (KASI), 776 Daedeokdae-ro, Yuseong-gu, Daejeon 305-348, Republic of Korea. \\ 2 Indian Institute of Astrophysics, Kormangala (IIA), Bangalore 560034, India. \\ 3 Aryabhatta Research Institute of Observational Sciences (ARIES), Nainital 263129, India. \\ 4 University of Science 85 Technology, 176 Gajeong-dong, Yuseong-gu, Daejeon, Republic of Korea \\ 5 Pt. Ravishankar Shukla University, Amanaka G.E.Road, Raipur, Chhatisgarh, India - 492010
}

Accepted-

\begin{abstract}
Bright-rimmed clouds form on the edges of H II regions affected by the high energy radiation from a central ionizing source. The UV radiation from the ionizing source results in compression and ionization causing either cloud disruption or further star formation. In this work, we present $\mathrm{R}$-band polarization measurements towards four bright-rimmed clouds, IC1396A, BRC 37, BRC 38, and BRC 39, located in the different directions of the H II region, Sh2-131, in order to map magnetic fields (B-fields) in the plane of the sky. These BRCs are illuminated by the O star HD 206267 and present a range of projected on sky geometries. This provides an opportunity to understand the magnetized evolution of BRCs. The B-field geometries of the clouds deduced from the polarization data, after correction for foreground ISM contamination, are seen to be connected to the ambient B-fields on the large scale. They seem to play an important role in shaping the cloud IC1396A and BRC 37. BRCs 38 and 39 show a broader and snubber head morphology possibly due to the B-fields being aligned with incoming radiation as explained in the simulations. A good general agreement is noted on comparing our observational results with the simulations supporting the importance of B-fields in BRC evolution. This work is the first step towards systematic mapping the B-fields morphology in multiple BRCs in an expanding H II region, extending the work presented by Soam et al. (2017b).
\end{abstract}

Key words: ISM: Globule; polarization: dust; ISM: magnetic fields; stars: emissionline

\section{INTRODUCTION}

The H II regions are formed when a massive star photoionizes and thus expand the surrounding medium. The formation of over-pressure ionozed bubbles are formed by the massive stars (OB-type) emitting the high energy photons (with rates of $\left.10^{47}-10^{50} \mathrm{~s}^{-1}\right)$. The shocks driven from the ionizing source results into the expansion of these bubbles. When the shock is isothermal, a thin, dense and generally unstable gas and dust shell forms at the H II region boundary. These boundaries are often found with peculiar and highly irregular structures. According to their structural appearances, these objects are named as fingers or pillars, speck globules, bright-rimmed clouds (BRCs), cometary globules (CGs) and elephant trunks (Leung, 1985). It was unclear whether these irregular structures are formed as a result of flow instabilities (Spitzer, 1954; Giuliani, 1979; Garcia-Segura \& Franco, 1996; Williams, 1999) or preexisting dense structures from the ambient interstellar medium advected through the ionization front (Reipurth, 1983). But recently Kinnear et al. (2014, 2015) have systematically investigated these objects and revealed that an initially ellipsoidal and inclined molecular cloud to an ionizing source could form most of the observed irregular structures from a filament and asymmetrical BRCs to a horse-head etc, by changing the initial density, geometry, inclination angle and the strength of the ionization flux. This work, combined with the previous investigation by Miao et al. (2009) on the formation mechanism of symmetrical type $\mathrm{A}, \mathrm{B}$ and $\mathrm{C}$ type $\mathrm{BRCs}$, provided a completed set

^ email:archanasoam.bhu@gmail.com, archana@kasi.re.kr 
mechanisms for the formation of various structures found on the boundaries of H II regions.

The radiation from the massive stars drives an implosion into the globules sitting in the vicinity. The radiative driven implosion (RDI) mode presupposes a rather structured ambient medium to which the H II region expands rapidly in directions where the density is relatively low. As this Rapid-type (R-type) ionization front encounters a relatively high density globule, it translates to a Delayed-type (D-type) and drives a convergent shock into the globule. Maximum compression of the globule occurs in this initial phase causing it to implode. Existing simulations of RDI focused on the onset and the efficiency of triggered star formation in isolated pre-existing clumps (Bisbas et al., 2011; Haworth et al., 2012), and on the driving of turbulence. The accretion luminosity of the embedded protostar in the globules could be increased as a result of the enhanced accretion rate due to strong compression during RDI process. But, on the other hand the luminosity can be decreased in the RDI process due to the photoevaporation of the parent core causing a decrease in the core mass (Motoyama et al., 2007). For investigating this process in detail and to model the collapse of the cores exposed to ultraviolet (UV) radiation from massive stars, Motoyama et al. (2007) presented some numerical simulations and estimated the mass loss rate dependence on the initial density profiles of cores and variation of UV fluxes. Their study also derived the simple analytic estimates of the accretion rates and final masses of the protostars.

First 3-D magnetohydrodynamical (MHD) simulations towards magnetized globules were presented by Henney et al. (2009). They found that photoevaporating globules will evolve into more flattened sheet like structures compared to the non-magnetic cases when the cloud initially has a strong B-field (i.e. 100 times the thermal pressure) perpendicular to the UV radiation direction. Later, they extended this work by adding B-fields of various strengths and orientations (Mackey \& Lim, 2011). One of the important results they obtained was that an initially perpendicular but weak and medium strength B-fields are finally swept away and get aligned with the pillar during its dynamical evolution. This is consistent with the observed field orientations in M16 (Sugitani et al., 2007) and in some CGs (e.g., Hodapp, 1987; Sridharan et al., 1996). However, in CG 3031 (BRC 51), Bhatt (1999) found that the projected B-fields are oriented perpendicular to the tail of the globule. Above results obtained on CG $30-31$ are different from the previous polarization studies towards various H II regions like RCW41 (Santos et al., 2012), NGC3576 (Shih et al., 2009) and Sh 156 (King \& Scarrott, 1983) where the B-fields are found to be parallel to the tail part. So far very few attempts have been made observationally to map the B-field geometry towards some BRCs such as BRC 20 (Targon et al., 2011), BRC 51 (CG 30-31, Bhatt 1999), BRC 74 (Kusune et al., 2015) and BRC 89 (Santos et al., 2014).

IC1396 is a young and active H II regions in the Cep OB2 containing clustered OB stars (Schwartz et al., 1991). It appears that the expansion of this H II region has resulted into sweeping up a molecular ring of radius $\sim 12$ pc (Patel et al., 1995). This region contains 15 small globules which are found embedded with IRAS sources (Schwartz et al., 1991). The large scale dynamical study using CO molecular line observations has been presented by
Patel et al. (1995) and Weikard et al. (1996). Sugitani et al. (1991) and Froebrich et al. (2005) found a rich population of BRCs and CGs seen in silhouette against the emission nebulae residing on the large molecular shell surrounding the H II region. The dominant source of UV radiation in $\mathrm{H}$ II region IC1396 is a O6.5V star HD206267 (Walborn \& Panek, 1984; Patel et al., 1995). This star is the member of Trumpler 37, a young open cluster which is located at the center of the Cep OB2 association (Simonson, 1968). From the photometric observations by Garrison \& Kormendy (1976) and Simonson (1968), Patel et al. (1995) found 12 stars with spectral types earlier than B1. Clearly the hottest star HD206267 (O6.5) in the center is the primary source of ionizing UV radiation.

To understand the effects of B-fields in the structural evolution of the clouds, it is important to the perform a systematic polarimetric study by selecting an H II region containing multiple BRCs at different locations, with known properties of ionizing source(s). Relatively closer regions (with less foreground extinction) with less complex geometry would be easier to study systematically. Assuming a preferred orientation of B-field prior to the formation of a $\mathrm{H}$ II region, the clouds in the different directions would show different magnetized evolution because of the different orientation of ionizing radiation. Soam et al. (2017b) have chosen Sh 185 region with IC 63 and IC 59 nebulae in different direction of the radiation from ionizing source $\gamma$ Cas but there were only two nebulae studied in that region. In the present study, we show the polarization results of four BRCs associated to IC1396 H II region. We have chosen these BRCs in north (BRC 38 or IC1396N), south (BRC 37), east (BRC 39) and west (IC1396A and BRC 36) directions of this H II region.

This study will give us an insight into the magnetized evolution of the multiple BRCs associated to the H II region as a function of the direction of the ionizing radiation. This paper presents the data acquisition information in section 2 . Results of the polarimetric observations are shown in section 3. In section 4, we discuss and analyze the results obtained and further conclude our findings in sections 5 .

\section{DATA ACQUISITION}

The polarization measurements towards IC1396 HII region were obtained on ten nights from October 2013 to January 2014 (see Table 1) using Aries IMaging POLarimeter (AIMPOL; Rautela et al., 2004) at $1 \mathrm{~m}$ optical telescope situated in Aryabhatta Research Institute of Observational Sciences (ARIES), India. The mean exposure time per observed frame per half wave plate (HWP) angle was $\sim 250$ sec for obtaining good signal-to-noise ratio. We obtained seven frames on each position of the HWP in one field and repeated the procedure for covering the four BRCs in IC1396 region. The details of the instrument and the procedure of data reduction for obtaining polarization values from observed data has been given in our previous papers (Soam et al., 2013, 2015, 2017b). Care has been taken in removing the instrumental polarization from the measurements by considering a constant value observed in various studies using this instrument. This value has been reported as $0.1 \%$ (see Soam et al., 2013, 2015; Neha et al., 2016). We also observed the polarized standard stars (results shown in Table 2) from Schmidt et al. 
Table 1. Log of polarimeteric observations towards IC1396 region in $R_{\mathrm{kc}}$ filter $\left(\lambda_{\mathrm{eff}}=0.760 \mu \mathrm{m}\right)$.

\begin{tabular}{ll}
\hline Cloud ID & Date of observations (year, month,date) \\
\hline IC 1396 & 2013, Oct, 29; 2013, Nov 08, 09, 10, 26, 28 \\
& 2013, Dec, 01, 03; 2014, Jan 03, 05 \\
\hline
\end{tabular}

Table 2. Results of observed polarized standard stars.

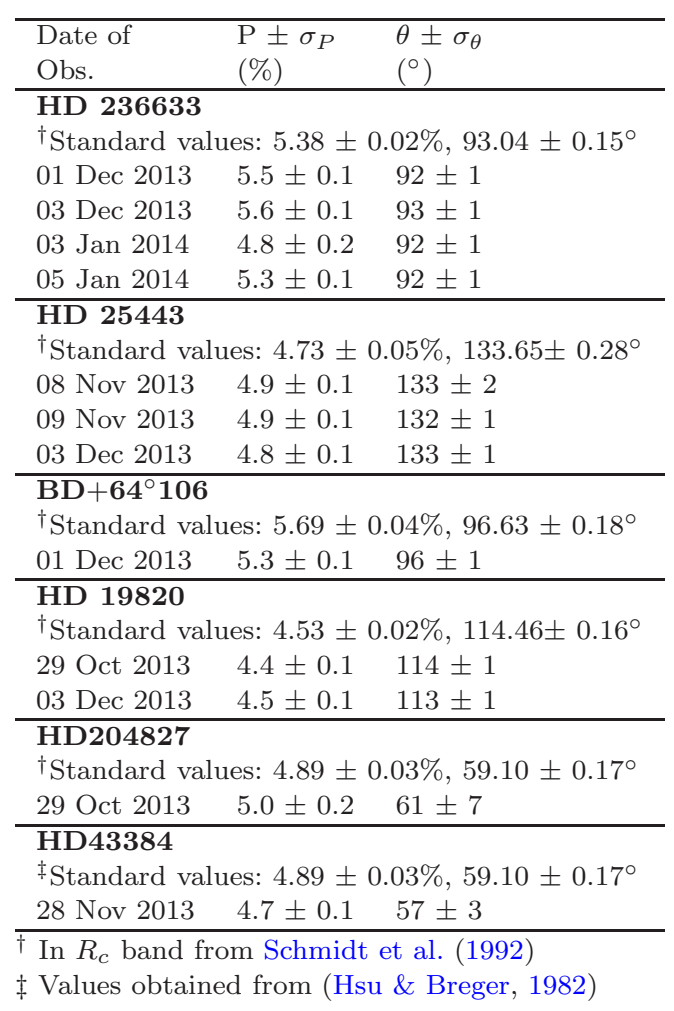

(1992) to determine the reference direction of the polarizer. A good correlation has been noticed among the observed values and the standard values given in Schmidt et al. (1992). These values were used to obtain the zero point offset correction which was applied later on the observed position angles of the target stars.

\section{RESULTS}

The results of our optical polarization measurements towards the stars projected on the fields of IC1396A (a.k.a. to elephant trunk nebula), BRC 37, BRC 38 (a.k.a. IC1396N) and BRC 39 are presented in this work. The vectors corresponding to 374 polarization measurements, are overlaid on $\mathrm{WISE}^{1} 12 \mu \mathrm{m}$ image containing these fields in Fig. 1. The expanding circular H II region IC1396 is clearly perceptible in the image. The direction of ionizing radiation and the position of ionizing source are also shown. The mean values of polarization measurements towards four BRCs are given in Table 3. The location of these clouds are shown in the zoomed images in north (BRC 38), south (BRC 37), east (BRC 39) and west (IC1396A). The orientation of Galactic

1 WISE image courtesy of NASA, JPL/Caltech, WISE Team
Table 3. Mean values of polarization results obtained towards BRCs studied in this work.

\begin{tabular}{lllr}
\hline Object & No. of stars & $\begin{array}{l}\mathrm{P} \pm \sigma_{\mathrm{P}} \\
(\%)\end{array}$ & $\begin{array}{r}\theta \pm \sigma_{\theta} \\
\left({ }^{\circ}\right)\end{array}$ \\
\hline IC1396A & 173 & $2.0 \pm 1.1$ & $53 \pm 18$ \\
BRC 37 & 40 & $1.6 \pm 0.8$ & $98 \pm 50$ \\
BRC 38 & 121 & $2.7 \pm 1.2$ & $51 \pm 17$ \\
BRC 39 & 40 & $1.8 \pm 1.0$ & $50 \pm 21$ \\
\hline
\end{tabular}

plane is shown with dashed white line at a position angle of $65^{\circ}$. Black open circle on the image shows the field of view $\left(8^{\prime}\right.$ diameter) of the instrument (AIMPOL) used for the observations. In our study we mapped the B-field geometry of the outer low density parts of the clouds. The distribution of degree of polarization $(\mathrm{P})$ and position angle $\left(\theta_{\mathrm{P}}\right)$ towards the four BRCs observed in IC1396 are shown in Fig. 2. We segregated the known YSOs, emission line sources, known binary systems and the normal stars from our observed sample based on the information from Simbad and literature. YSOs are identified in the vicinity of IC1396A (Sicilia-Aguilar et al., 2005, 2006), BRC 37, BRC 38 (Choudhury et al., 2010) and BRC 39.

\subsection{Polarimetric results of the YSOs found in the sample}

YSOs are found to show intrinsic polarization which is attributed to the asymmetric distribution of circumstellar material either as a disk or a flattened envelope (Breger \& Dyck, 1972; Bhatt, 2011). Therefore, we separated the polarization measurements of YSOs from the target stars. Out of the observed sources towards IC1396, 19 stars are identified as YSOs in Simbad. Hence we have polarization measurements of 19 YSOs towards this region out of which seven stars are projected on IC1396A (Sicilia-Aguilar et al., 2005, 2006) and twelve stars are located towards BRC 38 (Choudhury et al., 2010). The mean values of $\mathrm{P}$ and $\theta_{\mathrm{P}}$ with their standard deviations towards these YSOs are estimated to be $1.2 \pm 1.1 \%$ and $55 \pm 44^{\circ}$ in IC1396A and $2.4 \pm 1.2 \%$ and $46 \pm 24^{\circ}$ towards BRC 38 , respectively.

\section{DISCUSSION}

\subsection{Distance of IC1396 H II region}

IC1396 is found to be at $\sim 750$ pc by Garrison \& Kormendy (1976) using photometric and spectroscopic observations. It is a large ( $>2$ degree) and evolved H II region ionized by HD 206267 located near its center. HD206267 lies in Trumpler 37 cluster which is located in the Cep OB2 association at a distance of about 800 pc. Contreras et al. (2002) estimated distance to Trumpler 37 as $870 \pm 80$ pc. Majority of the studies towards this H II region assume a distance of $\sim 750 \mathrm{pc}$ to the clouds associated to this region. We have adopted a distance of $\sim 750 \mathrm{pc}$ (Garrison \& Kormendy, 1976) to this $\mathrm{H}$ II region for our further analysis.

We measure the polarization of stars background to the cloud for mapping the sky component of the B-fields in that cloud but it is very important to consider the polarization contribution of the foreground dust in the line of sight to obtain the intrinsic polarization solely from the dust in the 


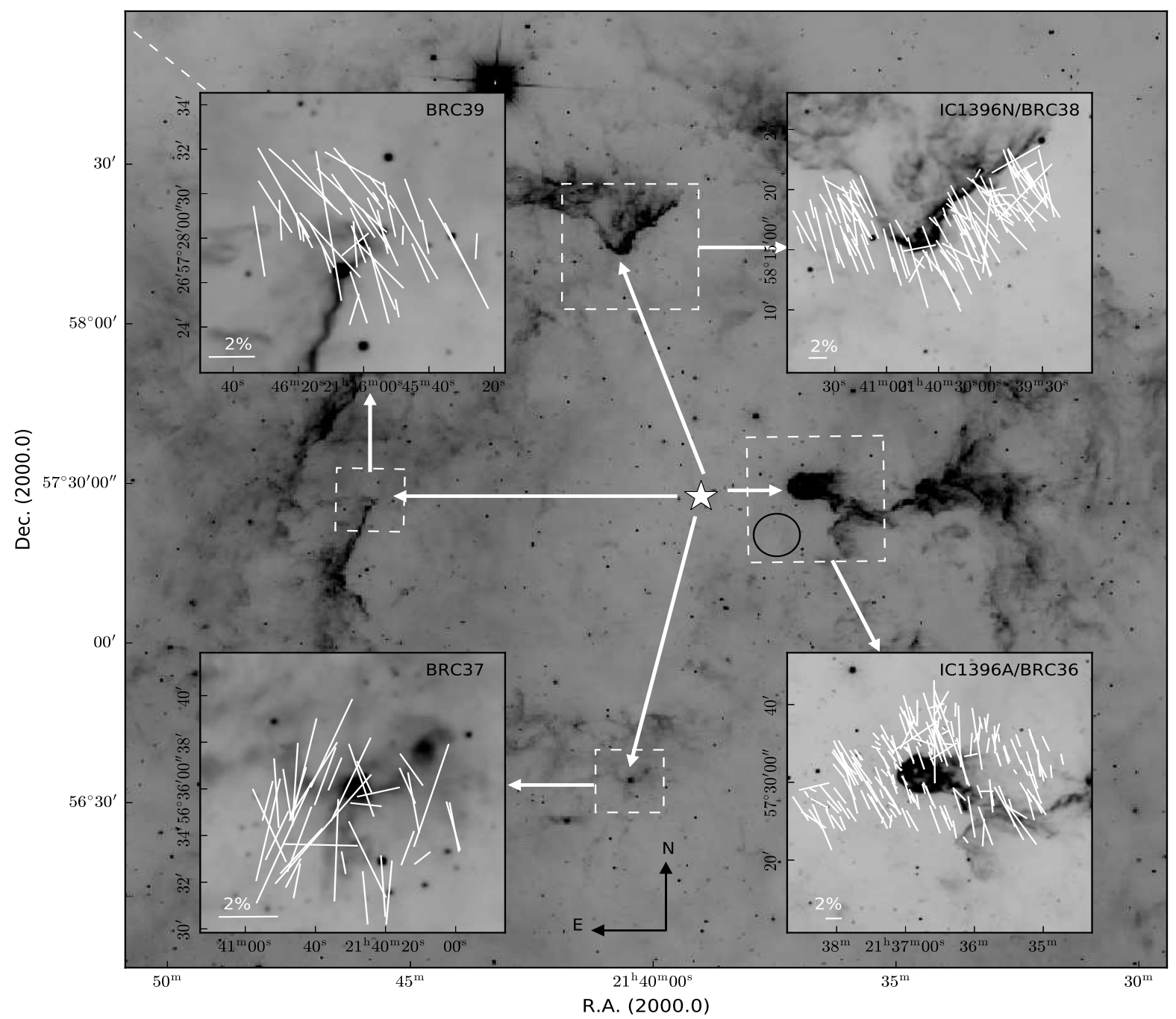

Figure 1. Optical polarization vectors over-plotted on WISE $12 \mu \mathrm{m}$ image of IC1396 H II region. The structures IC1396 A, BRC 37 , BRC 38 and BRC 39 with the B-field geometry are shown in zoomed-in images. Line segments with $2 \%$ polarization are shown for scaling. A white dashed line shows the position angles of the Galactic plane. The black circle in the dashed panel on the center right fo the image shows the field of view of the instrument (AIMPOL). Position angles are measures from north increasing towards east. The north and east directions are shown in the figure.

cloud. This contribution can vary depending on the distance of the cloud. For example, this contribution has not been found changing the measured values significantly as seen in various studies done on clouds located at distances less than 500 pc (e.g., Li et al., 2009; Soam et al., 2013). But it can affect the polarization measurements if there are relatively smaller $\mathrm{P}$ values $(\lesssim 1 \%)$ and the cloud distance is relatively larger $(\gtrsim 500 \mathrm{pc})$. Since the distance of the H II region studied here is $\sim 750 \mathrm{pc}$, we decided to remove the foreground polarization component added to the results by observing some stars located foreground to the region. Fig. 1 shows the uncorrected polarization vectors overlaid on IC1396 region.
Table 4. Polarization values of observed stars foreground to IC1396 region.

\begin{tabular}{llllll}
\hline Id & Star Name & $\begin{array}{l}\mathrm{V} \\
(\mathrm{mag})\end{array}$ & $\begin{array}{l}\mathrm{P} \pm \epsilon_{P} \\
(\%)\end{array}$ & $\begin{array}{l}\theta \pm \epsilon_{\theta} \\
\left({ }^{\circ}\right)\end{array}$ & $\begin{array}{l}\mathrm{D}^{\dagger} \\
(\mathrm{pc})\end{array}$ \\
\hline 1 & HD 207049 & 8.9 & $0.95 \pm 0.08$ & $5 \pm 2$ & 379 \\
2 & HD 206081 & 7.6 & $1.07 \pm 0.07$ & $64 \pm 1$ & 446 \\
3 & HD 209744 & 6.7 & $0.65 \pm 0.20$ & $55 \pm 8$ & 450 \\
4 & HD 210628 & 6.9 & $1.26 \pm 0.20$ & $60 \pm 4$ & 480 \\
5 & HD 239728 & 8.8 & $0.58 \pm 0.06$ & $115 \pm 3$ & 568 \\
6 & HD206267A & 5.6 & $1.02 \pm 0.07$ & $39 \pm 2$ & 606 \\
\hline
\end{tabular}

$\dagger$ The parallax measurements from Hipparcos (van Leeuwen, 2007) and GAIA Catalogues (TGAS; Gaia Collaboration, 2016) are used for the distance estimation.

\subsection{Subtraction of foreground polarization component}

The polarization caused by the material in ISM in a particular line of sight must be taken into account. Although 


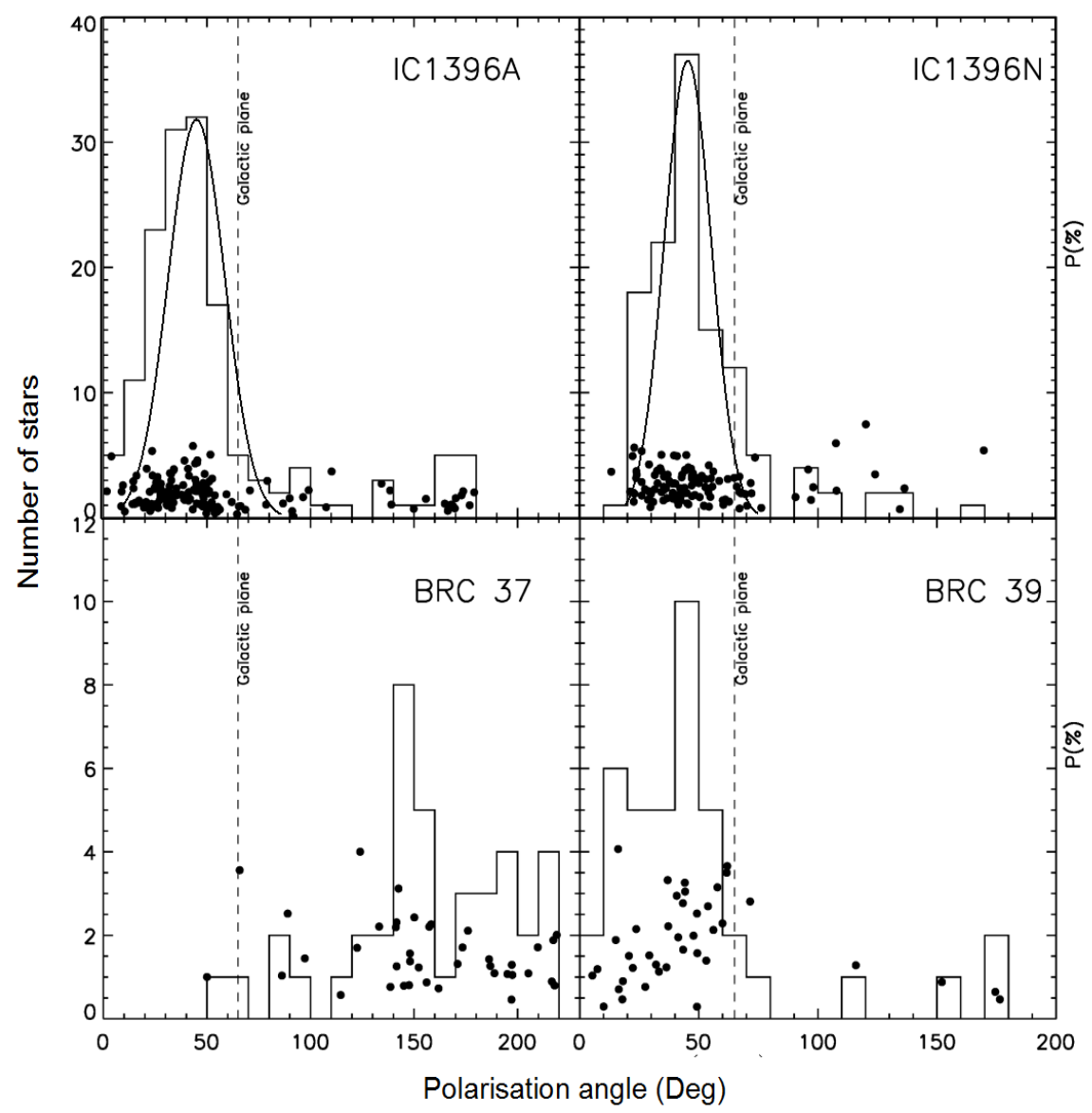

Figure 2. Plots of the $\mathrm{P}$ versus $\theta_{\mathrm{P}}$ of stars excluding peculiar stars projected on IC 1396A, BRC 37, BRC 38 (IC $1396 \mathrm{~N}$ ), and BRC 39. The histograms of the $\theta_{\mathrm{P}}$ with binsize $10^{\circ}$ are also presented. The position angles of the Galactic plane at the latitude of the clouds are shown using dashed linse.

we did not notice major change after the ISM contribution subtraction in the polarization results observed towards the cloud IC 63/59 (Soam et al., 2017b) at a distance of $\sim 200$ pc but we suspect that there may be a significant contribution of the ISM polarization in the results obtained towards IC1396 H II region due to its far location ( $\sim 750 \mathrm{pc})$.

For subtracting the interstellar polarization component from measured values, we searched for the stars that are located within a radius of $1^{\circ}$ around the center of IC1396 H II region with their available parallax measurements in Hipparcos (van Leeuwen, 2007) and GAIA ${ }^{2}$ Catalogues (TGAS; Gaia Collaboration, 2016). We only selected the normal stars excluding the peculiar sources such as emission line stars, stars in a binary or multiple system as per the information given in Simbad. The stars with the values of ratio between the parallax measurements and their uncertainties as $\geqslant 2$, have been considered for the analysis. Thus found six stars with their observed polarization results from this work, are listed in Table 4 with the ascending order of their distances. We also selected normal stars in available in $1^{\circ}$ radius from Heiles catalogue (Heiles, 2000) whose polarization values are available in the catalog. We have restricted these search of stars upto a distance of 800 pc. The variations of

2 http://www.esa.int the degree of polarization and position angle corresponding to foreground stars with their distances are shown in Fig. 3. The distribution of the Stokes parameters $q$ and $u$ with their distances is shown in Fig. 4. The dotted lines in the plot shows the distance of cluster Trumpler 37. In these figures, it is visible that there are two populations both in $q$ and $u$ which have different $\mathrm{P}$ and $\theta$ values at distances $\leqslant 300 \mathrm{pc}$ and greater than $300 \mathrm{pc}$. It might be possible that the some cloud at distance between 200-300 pc is responsible for the change in the polarization values beyond $300 \mathrm{pc}$. The stars within $300 \mathrm{pc}$ to $650 \mathrm{pc}$ are the immediate foreground to the clouds in IC1396 H II region. Therefore, we have used the observed values of the stars within $300 \mathrm{pc}$ to $650 \mathrm{pc}$ for correcting the foreground contribution.

To obtain the intrinsic polarization from the cloud dust only, we subtracted the polarization values of the foreground stars (shown in Table 4) from our measured values towards the clouds. For this purpose we followed the procedure with detailed discussion given in our previous papers (Soam et al., 2013, 2017a). We have overlaid the vectors with corrected polarization angles towards IC1396A, BRC 38, BRC 37 and BRC 39 in Fig. 5 and these values have been used for further analysis. 


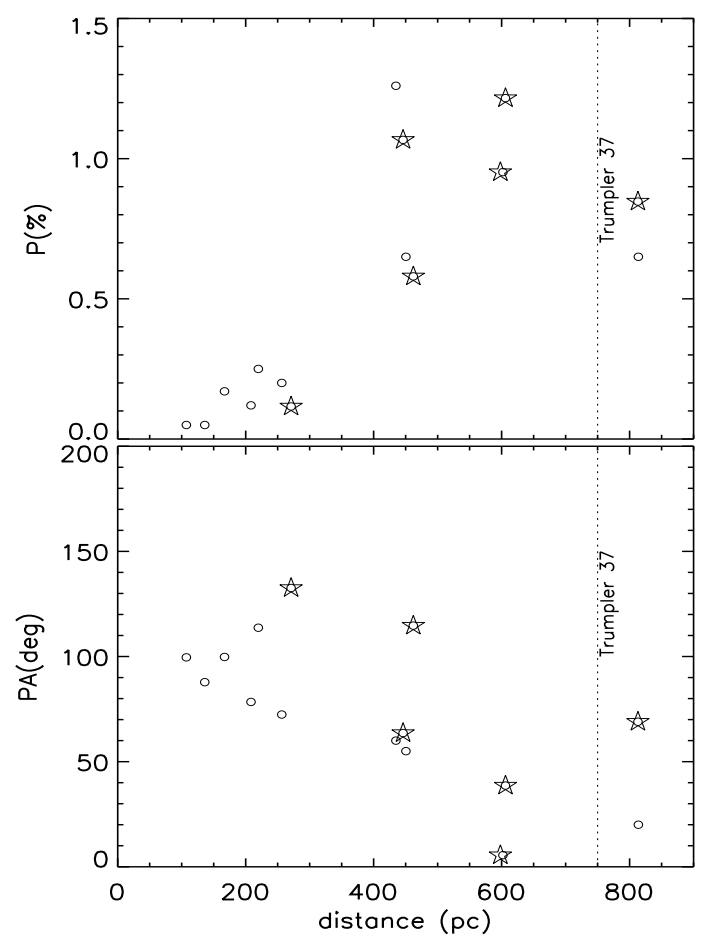

Figure 3. The change in the degree of polarization and the position angles with the distances of the foreground stars have been plotted in upper and lower panels, respectively. The open circles show the stars selected from Heiles catalogue (Heiles, 2000). The targets shown by stars are the foreground stars observed by us. The distance of these stars are taken from the Hipparcos (van Leeuwen, 2007) and GAIA Catalogues (TGAS; Gaia Collaboration, 2016). The dotted line represents the distance of cluster Trumpler 37.

\subsection{Magnetic field geometry}

We have carried out a systematic polarization study to map the B-fields in the H II region IC1396 by observing the multiple BRCs associated to this region. Fig. 6 shows the color composite image of IC1396 region made using WISE data. The image is labeled with the position of four BRCs in north, south, east and west directions. The position of the BRC 36 towards IC1396A, is shown using a box in cyan color at the position coincident to the peak $850 \mu \mathrm{m}$ peak emission (Morgan et al., 2008). The ionizing source HD206267 (spectral type $\mathrm{O} 6.5 \mathrm{~V}$ ) is also located in the image. The directions of ionizing radiations towards BRCs are also shown. The white line segments plotted on the BRCs represent the mean B-field orientation. The Strömgren sphere (Strömgren, 1939) created by ionizing radiation from HD206267 is shown by dashed circle. The mean value of the B-fields direction in these BRCs are inferred from the polarization vectors after correcting for the foreground component. We estimated the incident angles of the ionizing photons towards these BRCs by joining a line from the ionizing source HD 206267 radially to the positions of $850 \mu \mathrm{m}$ dust emission peaks in these clouds identified by Morgan et al. (2008). The directions of incident ionizing photons are estimated to be $90^{\circ}$ and $86^{\circ}$ in IC1396A and the globule BRC 36 located towards the tail of IC1396A, respectively. The angles of incident radiation towards BRC 37, BRC 38 and BRC 39 are found to be

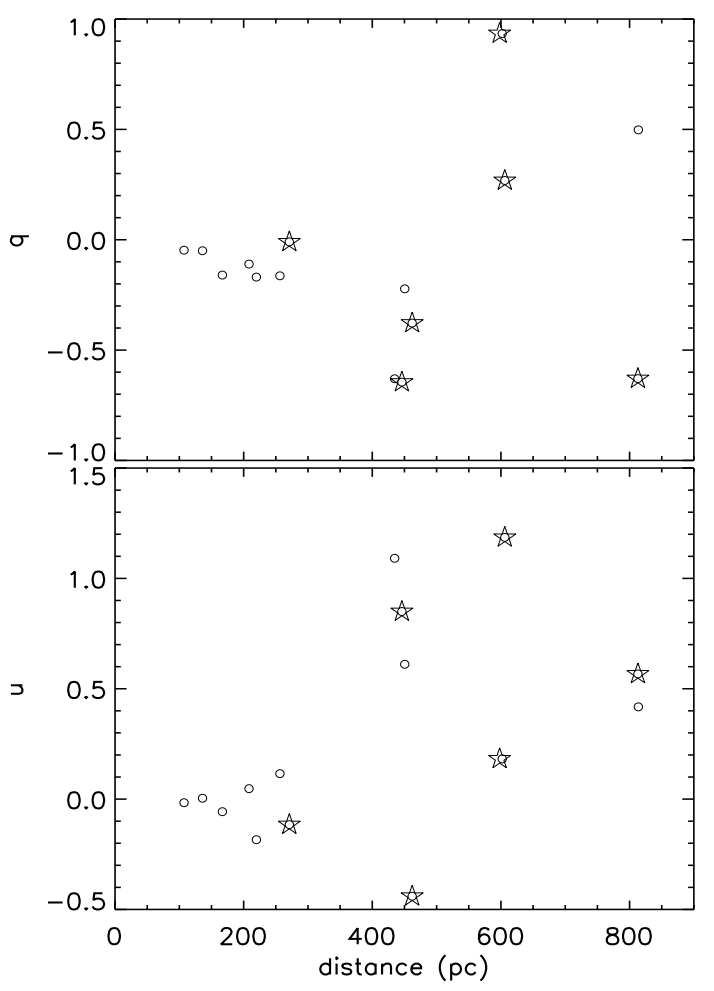

Figure 4. Same as Fig. 3 except for variations of Stokes parameters q (upper panel) and u (lower panel) with distances.

$\sim 160^{\circ}, \sim 30^{\circ}$ and $\sim 90^{\circ}$, respectively. Thus the projected offset angles between the incident ionizing radiation and the mean B-field orientation in BRC 37, BRC 38, IC1396 A and BRC 39 are estimated to be $\sim 10^{\circ}, \sim 20^{\circ}, \sim 35^{\circ}$ and $\sim 40^{\circ}$, respectively. These results imply that globally the B-fields are slanted w.r.t. the direction of the ionizing photons in the H II region IC1396. In BRC 37, the least value of the offset between the B-field direction and the ionizing photons suggest that the ambient B-fields are almost (though not exactly) parallel to the ionizing radiation. The inclination of the $\mathrm{B}$-fields and ionosing radiation in other three clouds are more than that in BRC 37. These results can be compared to the 3-D MHD simulations published by Henney et al. (2009) and Mackey \& Lim (2011) where the B-fields in the globules slanted w.r.t. the ionizing radiation are considered. The Bfields in these BRCs associated to H II region IC1396 (except BRC 37) are found to be parallel to the Galactic plane. This is in agreement with the results obtained by Novak et al. (2005); Li \& Henning (2011) in the studies of B-field morphology towards GMCs. This is also noticed in the results towards BRCs associated to Sh 2-185 region explained in Soam et al. (2017b).

To understand the magnetized evolution of the BRCs associated to H II region IC1396 in different directions and considering the MHD simulations by Henney et al. (2009), we made a cartoon diagram shown in Fig. 7. The left panel in the figure shows the H II region formed by gas, dust and ionized material swept by the high energy UV radiation from HD206267. The H II regions interact with the molecular clouds in the vicinity and pushes away the low density material faster than the high density cloud cores. In 


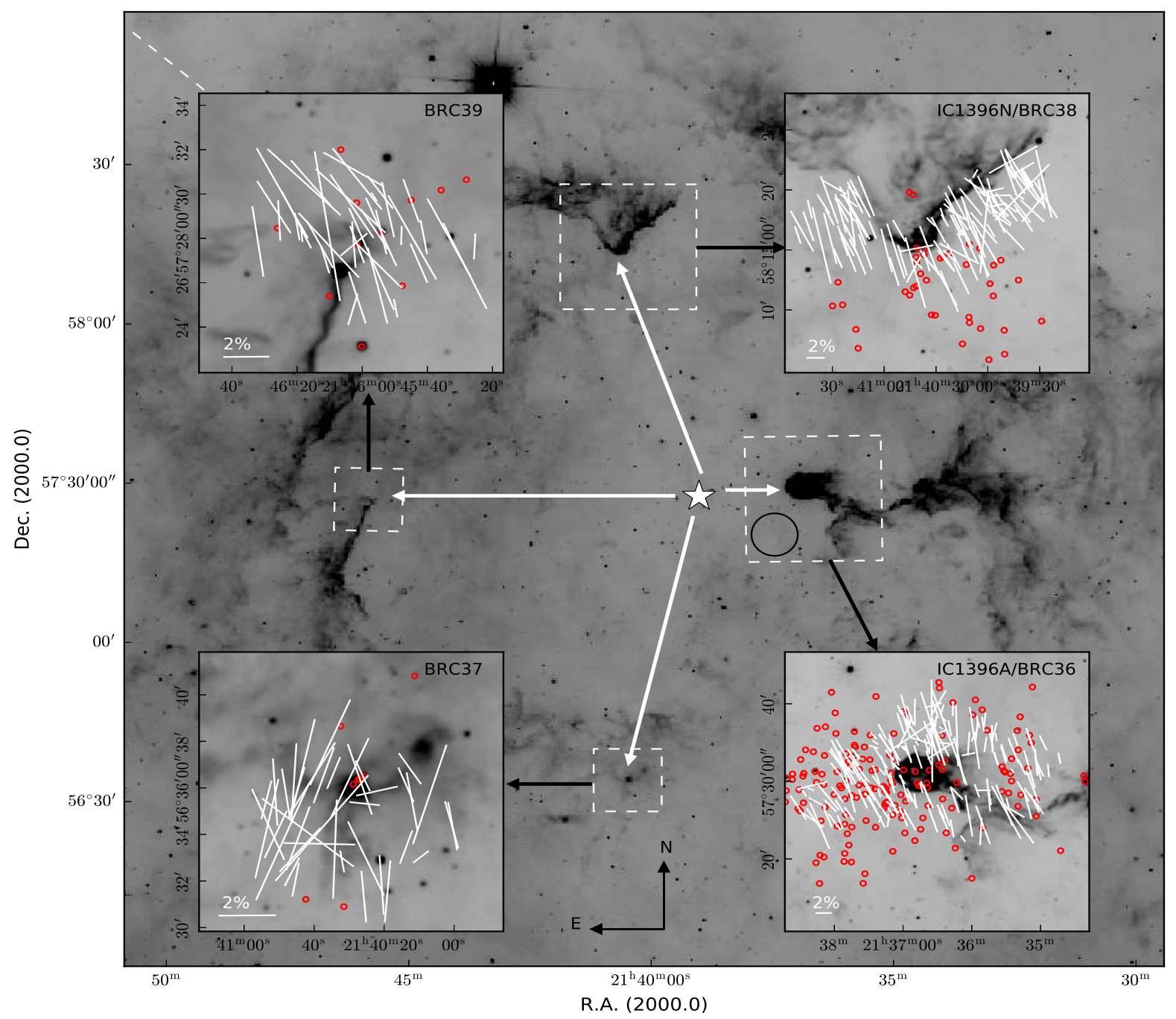

Figure 5. Polarization vectors (after subtracting the foreground polarization) overlaid on WISE $12 \mu \mathrm{m}$ images of IC1396A, BRC 37 , BRC 38 and BRC 39. Line segemnts with $2 \%$ polarization are shown to scale the value of P. The dashed line shows the position angle of Galactic plane at the latitude of the cloud. The positions of YSOs $\left(\mathrm{H}_{\alpha}\right.$ emission stars) distributed towards BRCs are shown using red open circles. Position angles are measures from north increasing eastward (directions shown in the figure).

Fig. 7, we showed the preexisting molecular clumps sitting on the edges of the H II region. The low density part of these clumps might have been ionized and pushed away by the expanding H II region giving rise to the bright rimmed structure. The ambient B-fields are shown with the dashed lines. In the right upper and lower panels, we have shown the fate of the two globules when the field lines are parallel and orthogonal to the ionizing radiation. The diagrams in right panels can probably explain the magnetized evolution of BRC 38 and IC1396A nebuale, respectively. The dynamical and kinematical motions in these BRCs due to RDI caused by UV radiation will be presented in our following work (Soam et al. in prep., Neha et al. under prep.) using various low and high density molecular gas tracers.

IC1396A globule in H II region IC1396 is also known as elephant trunk nebula. Such structures formed by pure RDI effect is composed of dense head at the front part followed by less dense tails (Lefloch \& Lazareff, 1994; Kessel-Deynet \& Burkert, 2000; Miao et al., 2009;
Bisbas et al., 2011; Haworth et al., 2012). IC1396A is appeared to be consistent to the RDI-triggered star formation scenario in a detailed study by Sicilia-Aguilar et al. (2014) using Herschel and Spitzer data. This is a much larger and more massive globule compared to the clouds modeled by Bisbas et al. (2011). Hence the self-gravity in IC1396A must play an important role in its dynamical evolution due to its higher mass $\left(\sim 200 M_{\odot}\right.$; Patel et al., 1995; Weikard et al., 1996). Morgan et al. (2004) found that the ionized boundary layer (IBL) pressure in this globule is relatively higher compared to the molecular pressure which creates an appreciable pressure imbalance in the cloud. This suggests that the cloud may be currently undergoing RDI process causing a triggered star formation in this globule (Sicilia-Aguilar et al., 2005).

Fig. 5 shows the foreground corrected polarization vectors representing the B-field geometry in IC1396A in zoomed-in panel. The offset of $\sim 35^{\circ}$ between the mean direction of B-field and the direction of ionizing radiation 


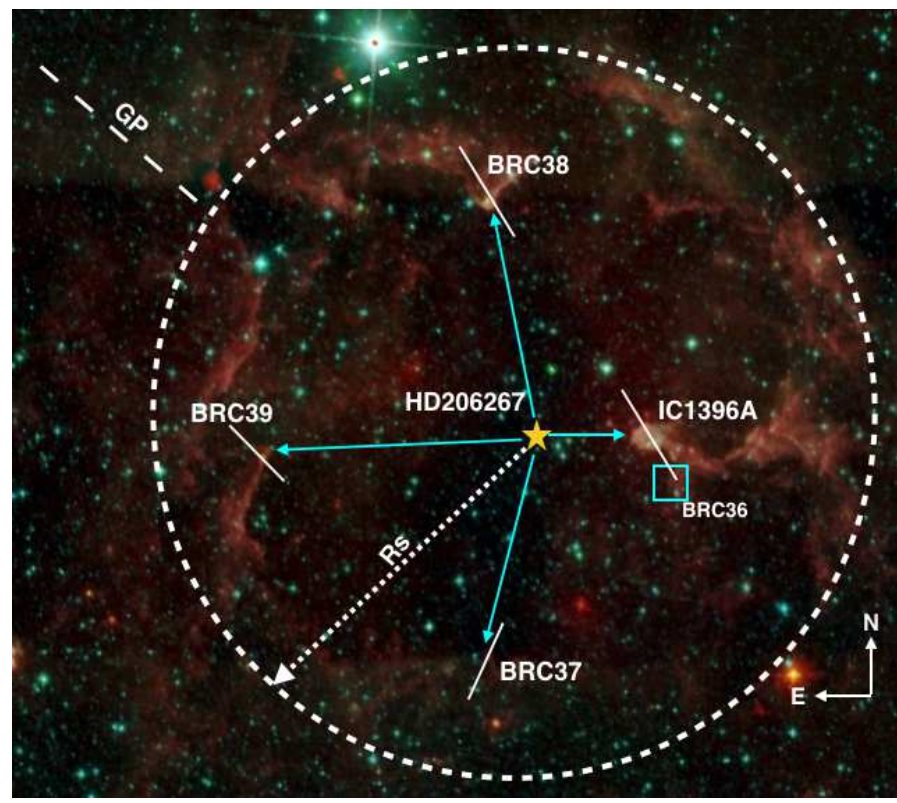

Figure 6. Figure shows the color composite image of IC1396 region made using WISE data. The image is labeled with the position of four BRCs in north, south, east and west. The position of the BRC 36 structure associated to elephant trunk nebula, is shown using a box in cyan color at the position of peak $850 \mu \mathrm{m}$ peak emission (Morgan et al., 2008). The ionizing source HD206267 (spectral type O6.5V) is also located in the image. The white line segments plotted on the BRCs represent the mean B-field orientation in these BRCs. The Strömgren sphere (Strömgren, 1939) created by ionizing radiation from HD206267 is shown by dashed circle.

suggests that the B-fields are slanted w.r.t. the direction of the incident photons from HD 206267. In IC1396A, we noticed that the degree of polarization in some of the sources lying in high density region (inference of the high and low densities are based on the WISE $12 \mu \mathrm{m}$ emission as described in Soam et al. (2017b)) is relatively low. The B-field observations in this cloud are matching with the MHD simulations performed by Henney et al. (2009) and Mackey \& Lim (2011) in the globules with strong initial B-fields slanted to the direction of ionizing radiation. In such cases, the globule acceleration caused by the rocket effect at the later stages $(0.12 \mathrm{Myr})$ is not radially away from the ionizing radiation. According to these simulations, the globule is swiftly flattens along its longer axis when the B-fields are strong and found slanted to the direction of ionizing radiation. In this case, the tail part of the cloud is shadowed and the surrounding material is accreted along the inclined field lines.

The distribution of YSOs ( $\mathrm{H} \alpha$ emission stars) towards the BRCs studied here are shown using the red circles in Fig. 5. The distribution of YSOs correlate with the masses of the clouds suggesting more number of YSOs in more massive cloud which is relatively closer to the ionizing source. The importance of B-fields in the evolution of dense cores and subsequent star formation can be better understood by probing the field geometries using NIR and submm polarimetric techniques. This work is only limited to investigate the B-fields in the low density regions of the cloud.

Sicilia-Aguilar et al. (2005) and Sicilia-Aguilar et al.
(2006), using deep Spitzer photometry, optical photometry and spectroscopy, found a population of $>200 \mathrm{H} \alpha$ emission stars with a mixed age of $\sim 4$ Myr old stars in central Trumpler 37 cluster and $\sim 1$ Myr old stars on the front arc of IC1396A nebula. In addition to this, some obscured IR-exess stars were found embedded in the cloud. Morales-Calderón et al. (2009) reported presence of several embedded Class I/II YSOs in this cloud. Twenty-four low mass and high accretion rate T-Tauri stars were identified by Barentsen et al. (2011). By investigating the distribution of these stars with different age and distances from the ionizing source, above studies proposed that the formation of the stellar population located in front of IC1396A nebula, had been triggered by the high energy radiation from HD 206267. This finding has been supported by the presence of hundreds of YSOs concentrated within and surroundings of the nebula revealed by the high sensitivity optical and mid-infrared surveys. IC1396A has a young star LkH $\alpha 349 \mathrm{c}$ in the head part. The outflows from the star LkH $\alpha 349 \mathrm{c}$ has created a cavity of $\sim 0.3 \mathrm{pc}$ in the globule head. This cavity is seen in IRAC $8 \mu \mathrm{m},{ }^{12} \mathrm{CO}(\mathrm{J}=1-0)$ and ${ }^{13} \mathrm{CO}(\mathrm{J}=1-0)$ maps by Nakano (1989), and the extinction maps obtained from the 2MASS data by Reach et al. (2009) suggesting that the globule is being reshaped from inside.

The foreground corrected polarization vector revealing the B-field structure in BRC 37 is shown in Fig. 5. The globule axis is almost aligned (not exactly) with the incident UV radiation. This suggest that the high energy radiation from HD 206267 might have affected the formation and evolution of this cloud (Williams et al., 2001; Fukuda et al., 2002; Miao et al., 2006). The mean direction of the B-field is aligned with the longer axis of the cloud. The least value of the offset $\sim 10^{\circ}$ (among the BRCs studied in this work) between the ionizing radiation and the B-field direction suggests that the initial B-fields are nearly parallel to the UV radiation. The curvature in the cloud's rim might have been caused by the radiation. The alignment of B-fields geometry of the cloud longer axis suggests that the B-fields could be dragged away from the ionizing source. The B-fields will eventually be aligned with the direction towards the exciting star i.e. the cloud longer axis if the B-field is frozen into the gas (e.g., Bertoldi, 1989). The structure of the B-fields in BRC 37 globule is similar to the B-field morphology found in M16 pillar by Sugitani et al. (2007).

Hydrodynamical simulation towards pillar like structure such as M16 is presented by Williams et al. (2001). These simulations suggest that the narrow pillar like structure will often occur in the H II regions with the largescale inhomogeneities and their evolution may be resulting into the extended sequences of radiation-induced star formation. The mass of shocked molecular gas at the head of the column like structure is interestingly found closer to the Jeans mass (Williams et al., 2001). BRC 37 is not in pressure equilibrium between the IBL pressure and the molecular pressure (Morgan et al., 2004) and found with a star IRAS 21388+5622 associated with outflows (Schwartz et al., 1991) and age 0.3 Myr (Duvert et al., 1990) embedded on the tip of the cloud. The formation of this star might have been triggered by the ionizing radiation. The identification of other near-infrared sources (Sugitani et al., 1995) nearly aligned with this star and the globule axis suggest the sequential star formation. This cloud is also found to be con- 


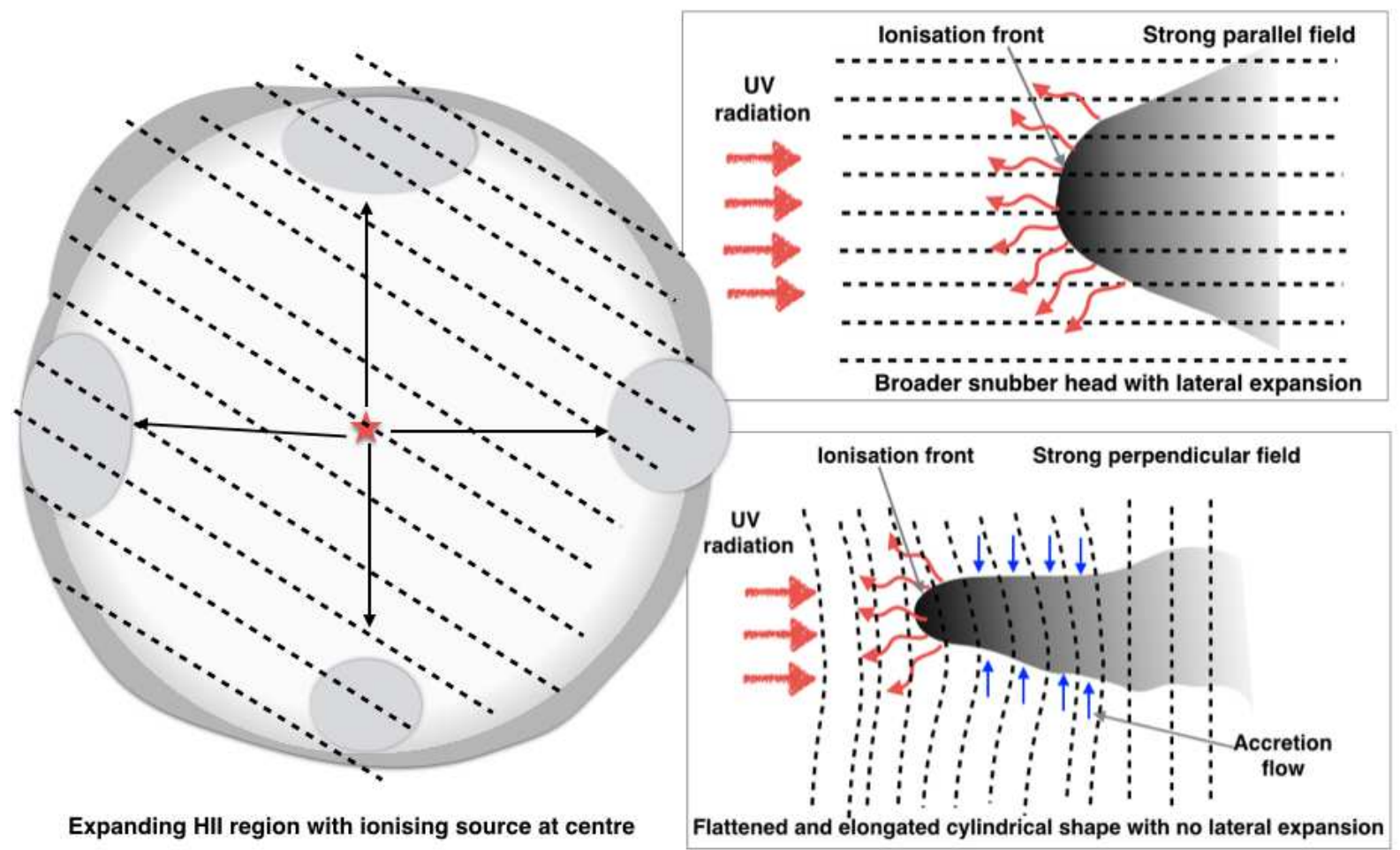

Figure 7. Schematic diagram showing the formation and expansion of H II region due to UV radition from central ionizing source. The formation of BRCs are understood by assuming the pre-existing clumps/clouds on the periphery of Strömgren sphere. The fates of molecular clouds in the presennce of parallel and perpendicular B-fields are also shown in the right column.

taining a young brown dwarf candidate which is reported as the first observational evidence of triggered brown-dwarf formation in BRCs (Ikeda et al., 2008).

The B-field geometry inferred from our polarization studies towards BRC 38 is also shown in Fig. 5. This cloud has the structural similarities with IC 63 globule associated to Sh 2-185 discussed in Soam et al. (2017b). We were not able to observe B-fields in the high density regions of the cloud BRC 38 using optical polarization technique. Thus the polarization map in Fig. 5 shows the B-field geometry on the periphery of the cloud. The offset of $\sim 20^{\circ}$ between the direction of the ionizing UV radiation and the mean direction of the B-field in the cloud suggests a slanted B-field geometry w.r.t. the ionizing radiation. In BRC 38 , particularly two features are noticeable. One is that the field lines exhibit two components on the western face of the globule which is looking towards the ionizing radiation. The other is that there is a density enhancement on the tip of the globule $(850 \mu \mathrm{m}$ SCUBA map; Morgan et al., 2004). This may be caused by the accumulation of the material due to the ionizing pressure. The mean direction of B-field is almost consistent to the mean B-field direction in IC1396A globule suggesting that the B-fields shown in these two clouds are well connected to the ambient large scale B-fields in the $\mathrm{H}$ II region IC1396. Henney et al. (2009) and Mackey \& Lim (2011) presented the 3D MHD simulations explaining the cases where initial B-fields are inclined (like found in BRC 38) to the incident ionizing radiation. In those cases, the lateral compression of the neutral globule is opposed by the B-fields and this results into a broader and snubber globule head with much simpler internal structure. These cases will not show any accretion on the globule tail part because thermal pressure of the ionized gas at the sides of the globule will not be sufficient enough to laterally compress the tails longitudinal B-fields. The photoevaporation flows are channeled by the B-fields towards the symmetry axis by means of a focusing shock (Henney et al., 2009). These simulation results are matching with our B-field observations in BRC 38.

BRC 38 is found to be undergoing triggered star formation due to the presence of high energy photons from the star HD206267 in the vicinity. IRAS21391+5802 is embedded on the tip of the nebula and associated with extended bipolar outflows (Sugitani et al., 1989). Beltrán et al. (2002), using millimeter wavelength observations revealed the other three embedded intermediate and low mass sources named as BIMA 1, 2 and 3 in this cloud. The young stars embedded in the cloud and associated with outflows indicate the ongoing star formation in the dense core at the tip of BRC 38 .

The B-field geometry towards BRC 39 is also shown in Fig. 5. The direction of radiation towards this cloud is at $\sim 90^{\circ}$. There is a clear bending of the B-fields on the head part of this cloud. These results are similar to the curved field geometries found in the studies towards LBN 437 (Soam et al., 2013) and B335 (Davidson et al., 2011). The mean direction of the B-field is slanted w.r.t. the ionizing radiation from HD 206267. There is a distinguishable elongated density enhancement feature on the lower rim of this cloud. Some of the vectors are found parallel to this feature suggesting that the material could be following the 
B-field lines in this region. By its structural axis orientation and morphology, BRC 39 is an asymmetrical type B cloud, its formation mechanism can be nicely illustrated by the scenario suggested in Kinnear et al. (2015) which indicates that it might have formed from an initially inclined prolate cloud under RDI effect.

Fig. 8 shows the schematics adopted from the simulation of Kinnear et al. (2015) to understand the modification in magnetic fields as found in BRC 39. The right most panel of the figure is a prolate cloud with its structure axis (major axis) inclined to the ionization radiation flux (shown by the red arrow). The global magnetic fields are shown using parallel green lines. After the cloud undergoes RDI process induced by ionizing radiation, the star-facing surface of the prolate cloud got shocked, and formed an elongated density enhancement thin layer with a highly condensed apex point at the top end, as shown in the rendered density image in the left panel of the figure. This case seems very similar to the observed features towards lower rim of BRC 39 as shown in the Fig. 5.

In Fig. 8, it is seen that the rear side of the cloud is not directly affected by the ionizing radiation and hence there is no significant change from its initial morphology therefore magnetic field there (green lines shown on upper part of the cloud) remains the original direction. On the other hand we can quantitatively analyse the effect on the magnetic fields on the ionizing star-facing side of the cloud (green lines shown on lower part of the cloud). In the right panel, the prolate cloud is initially embedded in the ambient magnetic field of the HII region. One of the green lines (the thicker one) can be taken as an example to show how it decomposes into two components, $\mathrm{B}_{\|}$and $\mathrm{B}_{\perp}$ to the ionization radiation coming from the direction denoted by the red arrow. After the RDI process, $B_{\|}$will not change but the previously perpendicular component $\mathrm{B}_{\perp}$ is curved into the shocked surface layer on the star-facing side (as described by the lower right panel of Fig. 7), as shown by the blue curve in the middle panel of the figure. The total magnetic field $\mathbf{B}$ is now approximately represented by the blue arrowed thick line in the middle panel, with an angle $\varphi$ from the original direction (thick green line). The final configuration of the magnetic field as seen in the lower part of BRC 39, is depicted by the parallel green lines as shown in the left panel of the figure which is bent from the direction of the original magnetic fields seen in upper part of the cloud.

BRC 39 is also found to be the cloud with ongoing star formation activity. The presence of embedded source IRAS $21445+5712$ is revealed with a far-infrared luminosity $\sim 96 \mathrm{~L} \odot$ (Panwar et al., 2014). Several H $\alpha$ emission stars associated to this cloud are reported by Ogura et al. (2002) and Nakano et al. (2012). The ongoing star formation activity is also suugested by the presence of water maser emission (Valdettaro et al., 2005) and two bright bow shocks $\mathrm{HH} 865 \mathrm{~A}$ and $\mathrm{HH}$ 865B emerging from IRAS $21445+5712$ (Froebrich et al., 2005)

\subsection{Magnetic field strength}

To compare the observation in this work and for understanding the role of B-fields in shaping the clouds in IC1396, we must know the field strength in the region. Fortunately, there are few prolific techniques available now to measure the field strength. One classical method to measure the strength of the B-field projected on to the plane of the sky was proposed by Chandrasekhar \& Fermi (1953). This technique was lately modified and new analysis methods are put forth to study the B-field and its interplay with turbulence (Ostriker et al., 2001; Falceta-Gonçalves et al., 2008). Using present observations, we estimated the strength of the B-fields using classical Chandersekhar \& Fermi formulation (Chandrasekhar \& Fermi, 1953). This formula requires the information of volume density $\left(\mathrm{n}_{\mathrm{H}_{2}}\right)$ in particles per cubic centimeter, the molecular line width in terms of FWHM $(\Delta \mathrm{V})$ in $\mathrm{kms}^{-1}$ and the dispersion in the measured polarization angle $(\delta \theta)$ in degree. The modified form of the formulations is given by Crutcher et al. (2004) which is

$$
\mathrm{B}_{\mathrm{pos}}=9.3 \sqrt{\mathrm{n}_{\mathrm{H}_{2}}} \frac{\Delta \mathrm{V}}{\delta \theta} \quad \mu \mathrm{G}
$$

The equipartition of kinetic and perturbed magnetic energies was assumed while deriving the original equation. Considering the polarization angle deviation and the velocity dispersion in the line of sight, the strength of the B-fields projected on the sky could be estimated.

The deviation in $\theta$ obtained by fitting Gaussian to the distribution of polarization angles is used to calculate the $\delta \theta$ in the clouds studied here. The dispersion in $\theta$ is then corrected by the uncertainty in $\theta$ using the steps given in Lai et al. (2001) and Franco et al. (2010). In this procedure, the dispersion is corrected in quadrature by the polarization angle using $\Delta \theta=\left(\sigma_{\mathrm{std}}{ }^{2}-\left\langle\sigma_{\theta}\right\rangle^{2}\right)^{1 / 2}$, where the mean error $\left\langle\sigma_{\theta}\right\rangle$ was calculated from $\left\langle\sigma_{\theta}\right\rangle=\Sigma \sigma_{\theta \mathrm{i}} / \mathrm{N}$, here $\sigma_{\theta \mathrm{i}}$ is the estimated uncertainty and $\sigma_{\text {std }}$ is the standard deviation in position angle (for details see Soam et al. (2017a)). We adopted the $\mathrm{CO}(\mathrm{J}=1-0)$ line FWHM from our ongoing molecular line survey of BRCs using Taeduk Radio Astronomical Observatory (TRAO; Soam et al. in prep.). We checked the perfect Gaussian profile of $\mathrm{CO}$ lines and estimated the average FWHM as $2.1 \pm 0.61 \mathrm{~km} \mathrm{~s}^{-1}$ and $1.51 \pm 0.07 \mathrm{~km} \mathrm{~s}^{-1}$ towards IC1396A and BRC 37, respectively. CO(1-0) observations towards SFO 38 were affected by the outflow activity from the embedded protostar hence we adopted the $\mathrm{C}^{18} \mathrm{O}(1-$ 0 ) line width which is found to be $1.31 \pm 0.10 \mathrm{~km} \mathrm{~s}^{-1}$ (Neha et al. under prep.). The line width of ${ }^{13} \mathrm{CO}(2-1)$ towards BRC 39 as $1.7 \mathrm{~km} \mathrm{~s}^{-1}$ (due to unavailability of CO data) has been adopted from Weikard et al. (1996). The volume densities toward these clouds are calculated using the hydrogen column density information from Morgan et al. (2010) which is found to be $16 \times 10^{21} \mathrm{~cm}^{-2}$ towards BRC 39. For other two clouds IC 1396A and BRC 38, we considered the average column density as $\sim 10^{22} \mathrm{~cm}^{-2}$ (based on the values given for all other BRCs in Morgan et al. (2010)). This value is found similar to the column densities obtained by $\mathrm{CO}(1-0)$ and $\mathrm{C}^{18} \mathrm{O}(1-0)$ observations (Soam et al. in prep., Neha et al. in prep.) using the relation given by Garden et al. (1991)). Using the only angular extents of the clouds where polarization observations are made and taking $750 \mathrm{pc}$ as the distance to IC $1396 \mathrm{H}$ II region, we estimated the volume densities of IC 1396A, BRC 38 and BRC 39 as $9.3 \times 10^{3} \mathrm{~cm}^{-2}$, $7.4 \times 10^{4} \mathrm{~cm}^{-2}$, and $3.8 \times 10^{4} \mathrm{~cm}^{-2}$, respectively. Using the volume density and line width information in CF relation, we estimated the magnetic field strengths as $\sim 110 \mu \mathrm{G}$, $\sim 220 \mu \mathrm{G}$, and $\sim 150 \mu \mathrm{G}$ towards IC1396A, BRC 38, and 


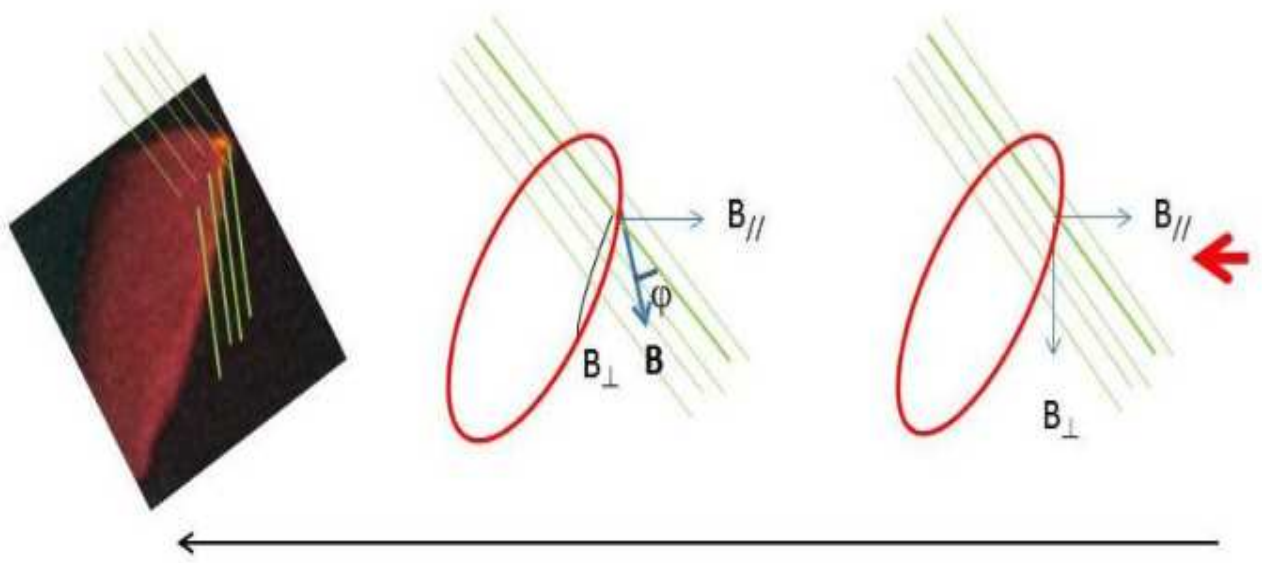

Figure 8. Scematics for depicting structural evolution of BRC 39 using the simulations from Kinnear et al. (2015). The evolutionalry track is shown from right to left represented by an arrow. Green segments show the global magnetic field lines and red arrow indicated the direction of ionizing radiation towards the cloud. Two orthogonal components of plane of the sky magnetic fields are labelled as $\mathrm{B}_{\|}$ and $\mathrm{B}_{\perp}$.

BRC 39, respectively. In case of BRC 38, we have used the polarisation values only on the tip of the cloud for estimating the magnetic field strength because we are adopting the $\mathrm{C}^{18} \mathrm{O}(1-0)$, optically thin tracer, line width in the calculation of B-field strength. Rest of the polarization measurements are mostly projected outside the cloud part therefore we did not use these values. B-field strength towards BRC 37 has not been estimated due to very high dispersion $\left(>25^{\circ}\right)$ in polarization angle which breaks one of the assumptions of the $\mathrm{CF}$ relation.

\subsection{Pressure budget in BRCs}

To compare and investigate the importance of various pressures in the BRCs studied here, we estimated the thermal $\left(\mathrm{P}_{\text {th }}\right)$, turbulent $\left(\mathrm{P}_{\text {turb }}\right)$, and magnetic pressures $\left(\mathrm{P}_{\mathrm{mag}}\right)$ towards these clouds. The IBL pressure $\left(\mathrm{P}_{\mathrm{IBL}}\right)$ values were adopted from Morgan et al. $(2004)^{3}$. The datasets characterized the physical properties of the IBL of the BRCs. They compared the measured flux $(\Phi)$ and predicted $\left(\Phi_{\mathrm{P}}\right)$ ionizing fluxes incident the rims of these clouds. The measured ionizing fluxes are found to be lesser than the predicted ionizing flux based on the spectral type of ionizing source and the distance of cloud from the source. Though the predicted ionizing fluxes can be considered as the upper limit because the loss in photons caused by absorption from intervening material between the star and the cloud has not been considered by Morgan et al. (2004) also the distance between star and the clouds are projected values. The values of pressures estimated towards BRCs studied here are shown in Table 5. Various pressures in the BRCs studied here, can be compared in the table. Dynamical pressure in the clouds is found to be lower than the external pressure which suggests that the shock driven by the photoionization could propagate into

3 They carried out a radio, optical and infrared wavelength imaging survey of 44 BRCs using the NRAO/VLA Sky Survey (NVSS), Digitised Sky Survey (DSS) and the Midcourse Space eXperiment (MSX) archived data the molecular cloud, causing it to implode and trigger star formation. Magnetic pressures of the clouds are estimated using the B-field strength over the region where optical polarimetry covers the cloud. Magnetic pressure is found to be lower than the external pressure in these BRCs except in BRC 38. The magnetic pressure in this cloud is estimated to be around two times the external pressure suggesting that fields can support cloud against external pressure.

\subsection{Polarization in YSOs}

The spectral type and $\mathrm{H} \alpha$ equivalent width (EW) for some of the YSOs observed in this work towards IC1396 are given by Sicilia-Aguilar et al. (2005, 2006), and Choudhury et al. (2010). The spherically distributed circumstellar dust around a central YSO producing infrared fluxes lead to a very large extinction along the line of sight at optical wavelength. Hence, the infrared emitting dust must be distributed in a flattened disk-like geometry (Strom, 1972). This can cause the high polarization when viewed from edgeon. Breger \& Dyck (1972) detected the intrinsic polarization in several pre-main sequence stars (PMS) in NGC 2264. The observed infrared excess emission, $\mathrm{H} \alpha$ emission and the intrinsic polarization of the YSOs suggest the presence of circumstellar disks around these objects. We found a higher polarization and $\mathrm{H} \alpha \mathrm{EW}$ associated to the stars with higher extinction. Some of the YSOs have low polarization even with the high extinction. The may be due to the dependence of polarization on viewing angle (edge on or face on).

\section{CONCLUSIONS}

We have made a first systematic study of B-fields towards multiple BRCs in different directions of H II region IC1396 at different distances from the same central ionizing source. The star, HD 206267 with spectral type O6.5V, located at a distance of $\sim 600 \mathrm{pc}$, is supposed to be the main ionizing source for H II region IC1396. B-field study of the BRCs in 
Table 5. Pressure estimation towards BRCs studied in this work. The values of IBL pressure are adopted from Morgan et al. (2004). The temperature information for estimating thermal pressure are obtained from Sicilia-Aguilar et al. (2014) towards IC1396A. The temeperature information towards BRC 37, BRC 38, and BRC 39 have been taken from Sugitani et al. (2000). In the table, IBL, thermal, turbulent and magnetic pressures denoted by $\mathrm{P}_{\mathrm{IBL}}, \mathrm{P}_{\text {th }}, \mathrm{P}_{\text {turb }}$, and $\mathrm{P}_{\mathrm{mag}}$, repectively.

\begin{tabular}{|c|c|c|c|c|c|c|}
\hline Object & $\begin{array}{l}\text { Measured Ionizing flux } \\
\Phi\left(10^{18} \mathrm{~cm}^{-2} \mathrm{~s}^{-1}\right)\end{array}$ & $\begin{array}{l}\text { Predicted ionizing flux } \\
\Phi_{\mathrm{P}}\left(10^{18} \mathrm{~cm}^{-2} \mathrm{~s}^{-1}\right)\end{array}$ & $\begin{array}{l}\mathrm{P}_{\text {IBL }} \\
\left(10^{-10} \mathrm{erg} \mathrm{cm}^{-3}\right)\end{array}$ & $\begin{array}{l}\mathrm{P}_{\mathrm{th}} \\
\left(10^{-10} \mathrm{erg} \mathrm{cm}^{-3}\right)\end{array}$ & $\begin{array}{l}P_{\text {turb }} \\
\left(10^{-10} \mathrm{erg} \mathrm{cm}^{-3}\right)\end{array}$ & $\begin{array}{r}P_{\mathrm{mag}} \\
\left(10^{-10} \mathrm{erg} \mathrm{cm}^{-3}\right) \\
\end{array}$ \\
\hline IC1396A & 2.4 & 59.6 & 1.0 & 0.05 & 1.7 & 0.6 \\
\hline BRC 37 & 2.0 & 10.2 & 15.0 & 0.12 & 2.6 & - \\
\hline BRC 38 & 2.8 & 13.3 & 11.0 & 0.05 & 1.0 & 21.0 \\
\hline BRC 39 & 1.9 & 9.2 & - & 0.08 & 2.1 & 3.2 \\
\hline
\end{tabular}

east, west, north and south give an idea of the complete geometry of the B-field in this region. To interpret the B-field geometry, we have subtracted the polarization contribution of ISM dust in the line of sight by observing the stars foreground to the clouds. The corrected results are used to interpret the B-field geometries. The orientations of mapped B-fields are found parallel to that of the Galactic plane suggesting a well connected field structure from larger scale to cloud scale. It seems that the fields lines in the BRCs studied here, were slanted to the direction of ionizing radiation prior to being affected by it. B-fields in BRC 37 and BRC 38 seem to be following the structure of the cloud. In IC1396A, may be due to the presence of strong B-fields, the structure of the globule becomes anvilled and elongated in shape. Our results are matched with the MHD simulations conducted towards BRCs to test their magnetized evolution. The fields lines are bent at the head part of BRC 39 . BRC 38 represents the case of strong B-fields almost parallel to the direction of ionizing radiation. The B-field strengths are estimated as $\sim 110 \mu \mathrm{G}, \sim 220 \mu \mathrm{G}$, and $\sim 150 \mu \mathrm{G}$ towards IC1396A, BRC 38, and BRC 39, respectively. The pressure budget of these BRCs are tested and it is found that the dynamical pressures in the clouds are lower than the external pressure which suggests that shock driven by the photoionization could propagate into the molecular cloud, causing it to implode and trigger star formation.

\section{ACKNOWLEDGEMENT}

This research has made use of the Simbad database, operated at CDS, Strasbourg, France. We also acknowledge the use of NASA's SkyView facility (http://skyview.gsfc.nasa.gov) located at NASA Goddard Space Flight Center. AS thanks KASI for the post-doctoral research fund. CWL was supported by Basic Science Research Program through the National Research Foundation of Korea (NRF) funded by the Ministry of Education, Science and Technology (NRF-2016R1A2B4012593). A.S. thanks Dr. Piyush Bhardwaj for the help during the observations.

\section{REFERENCES}

Barentsen G., et al., 2011, MNRAS, 415, 103

Beltrán M. T., Girart J. M., Estalella R., Ho P. T. P., Palau A., 2002, ApJ, 573, 246

Bertoldi F., 1989, ApJ, 346, 735

Bhatt H. C., 1999, MNRAS, 308, 40

Bhatt H. C., 2011, Pramana, 77, 19

Bisbas T. G., Wünsch R., Whitworth A. P., Hubber D. A., Walch S., 2011, ApJ, 736, 142
Breger M., Dyck H. M., 1972, ApJ, 175, 127

Chandrasekhar S., Fermi E., 1953, ApJ, 118, 113

Choudhury R., Mookerjea B., Bhatt H. C., 2010, ApJ, 717, 1067

Contreras M. E., Sicilia-Aguilar A., Muzerolle J., Calvet N., Berlind P., Hartmann L., 2002, AJ, 124, 1585

Crutcher R. M., Nutter D. J., Ward-Thompson D., Kirk J. M., 2004, ApJ, 600, 279

Davidson J. A., et al., 2011, ApJ, 732, 97

Duvert G., Cernicharo J., Bachiller R., Gomez-Gonzalez J., 1990, A\&A, 233, 190

Falceta-Gonçalves D., Lazarian A., Kowal G., 2008, ApJ, 679, 537

Franco G. A. P., Alves F. O., Girart J. M., 2010, ApJ, 723, 146

Froebrich D., Scholz A., Eislöffel J., Murphy G. C., 2005, A\&A, 432,575

Fukuda N., Hanawa T., Sugitani K., 2002, ApJ, 568, L127

Gaia Collaboration 2016, VizieR Online Data Catalog, 1337

Garcia-Segura G., Franco J., 1996, ApJ, 469, 171

Garden R. P., Hayashi M., Hasegawa T., Gatley I., Kaifu N., 1991, ApJ, 374, 540

Garrison R. F., Kormendy J., 1976, PASP, 88, 865

Giuliani Jr. J. L., 1979, ApJ, 233, 280

Haworth T. J., Harries T. J., Acreman D. M., 2012, MNRAS, 426,203

Heiles C., 2000, AJ, 119, 923

Henney W. J., Arthur S. J., de Colle F., Mellema G., 2009, MNRAS, 398, 157

Hodapp K.-W., 1987, ApJ, 319, 842

Hsu J.-C., Breger M., 1982, ApJ, 262, 732

Ikeda H., et al., 2008, AJ, 135, 2323

Kessel-Deynet O., Burkert A., 2000, MNRAS, 315, 713

King D. J., Scarrott S. M., 1983, MNRAS, 202, 11

Kinnear T. M., Miao J., White G. J., Goodwin S., 2014, MNRAS, 444,1221

Kinnear T. M., Miao J., White G. J., Sugitani K., Goodwin S., 2015, MNRAS, 450, 1017

Kusune T., et al., 2015, ApJ, 798, 60

Lai S.-P., Crutcher R. M., Girart J. M., Rao R., 2001, ApJ, 561,864

Lefloch B., Lazareff B., 1994, A\&A, 289, 559

Leung C. M., 1985, in Black D. C., Matthews M. S., eds, Protostars and Planets II. pp 104-136

Li H.-B., Henning T., 2011, Nature, 479, 499

Li H.-b., Dowell C. D., Goodman A., Hildebrand R., Novak G., 2009, ApJ, 704, 891

Mackey J., Lim A. J., 2011, MNRAS, 412, 2079

Miao J., White G. J., Nelson R., Thompson M., Morgan L., 2006, MNRAS, 369, 143

Miao J., White G. J., Thompson M. A., Nelson R. P., 2009, ApJ, 692,382

Morales-Calderón M., et al., 2009, ApJ, 702, 1507

Morgan L. K., Thompson M. A., Urquhart J. S., White G. J., Miao J., 2004, A\&A, 426, 535

Morgan L. K., Thompson M. A., Urquhart J. S., White G. J., 2008, A\&A, 477, 557

Morgan L. K., Figura C. C., Urquhart J. S., Thompson M. A., 2010, MNRAS, 408, 157 
Motoyama K., Umemoto T., Shang H., 2007, A\&A, 467, 657

Nakano T., 1989, MNRAS, 241, 495

Nakano M., Sugitani K., Watanabe M., Fukuda N., Ishihara D., Ueno M., 2012, AJ, 143, 61

Neha S., Maheswar G., Soam A., Lee C. W., Tej A., 2016, A\&A, 588, A45

Novak G., Griffin G. S., Krejny M., Li H., Loewenstein R. F., Newcomb M. G., Calisse P. G., Chuss D. T., 2005, in American Astronomical Society Meeting Abstracts \#206. p. 506

Ogura K., Sugitani K., Pickles A., 2002, AJ, 123, 2597

Ostriker E. C., Stone J. M., Gammie C. F., 2001, ApJ, 546, 980

Panwar N., Chen W. P., Pandey A. K., Samal M. R., Ogura K., Ojha D. K., Jose J., Bhatt B. C., 2014, MNRAS, 443, 1614

Patel N. A., Goldsmith P. F., Snell R. L., Hezel T., Xie T., 1995, ApJ, 447, 721

Rautela B. S., Joshi G. C., Pandey J. C., 2004, Bulletin of the Astronomical Society of India, 32, 159

Reach W. T., et al., 2009, ApJ, 690, 683

Reipurth B., 1983, A\&A, 117, 183

Santos F. P., Roman-Lopes A., Franco G. A. P., 2012, ApJ, 751,138

Santos F. P., Franco G. A. P., Roman-Lopes A., Reis W., RománZúñiga C. G., 2014, ApJ, 783, 1

Schmidt G. D., Elston R., Lupie O. L., 1992, AJ, 104, 1563

Schwartz R. D., Wilking B. A., Giulbudagian A. L., 1991, ApJ, 370,263

Shih Y. H., Chen W. P., Hsiao W. S., Tamura M., 2009, in Soonthornthum B., Komonjinda S., Cheng K. S., Leung K. C., eds, Astronomical Society of the Pacific Conference Series Vol. 404, The Eighth Pacific Rim Conference on Stellar Astrophysics: A Tribute to Kam-Ching Leung. p. 27

Sicilia-Aguilar A., Hartmann L. W., Hernández J., Briceño C., Calvet N., 2005, AJ, 130, 188

Sicilia-Aguilar A., Hartmann L. W., Fürész G., Henning T., Dullemond C., Brandner W., 2006, AJ, 132, 2135

Sicilia-Aguilar A., Roccatagliata V., Getman K., Henning T., Merín B., Eiroa C., Rivière-Marichalar P., Currie T., 2014, A\&A, 562, A131

Simonson III S. C., 1968, ApJ, 154, 923

Soam A., Maheswar G., Bhatt H. C., Lee C. W., Ramaprakash A. N., 2013, MNRAS, 432, 1502

Soam A., Maheswar G., Lee C. W., Dib S., Bhatt H. C., Tamura M., Kim G., 2015, A\&A, 573, A34

Soam A., Lee C. W., Maheswar G., Kim G., Neha S., Kim M.-R., 2017a, MNRAS, 464, 2403

Soam A., Maheswar G., Lee C. W., Neha S., Andersson B.-G., 2017b, MNRAS, 465, 559

Spitzer Jr. L., 1954, ApJ, 120, 1

Sridharan T. K., Bhatt H. C., Rajagopal J., 1996, MNRAS, 279, 1191

Strom S. E., 1972, PASP, 84, 745

Strömgren B., 1939, ApJ, 89, 526

Sugitani K., Fukui Y., Mizuni A., Ohashi N., 1989, ApJ, 342, L87

Sugitani K., Fukui Y., Ogura K., 1991, ApJS, 77, 59

Sugitani K., Tamura M., Ogura K., 1995, ApJ, 455, L39

Sugitani K., Matsuo H., Nakano M., Tamura M., Ogura K., 2000, AJ, 119, 323

Sugitani K., et al., 2007, PASJ, 59, 507

Targon C. G., Rodrigues C. V., Cerqueira A. H., Hickel G. R., 2011, ApJ, 743, 54

Valdettaro R., Palla F., Brand J., Cesaroni R., 2005, A\&A, 443,535

Walborn N. R., Panek R. J., 1984, ApJ, 286, 718

Weikard H., Wouterloot J. G. A., Castets A., Winnewisser G., Sugitani K., 1996, A\&A, 309, 581

Williams R. J. R., 1999, MNRAS, 310, 789

Williams R. J. R., Ward-Thompson D., Whitworth A. P., 2001, MNRAS, 327, 788 van Leeuwen F., 2007, A\&A, 474, 653 